\title{
Downregulation of T-cell lymphoma invasion and metastasis-inducing factor 1 induces cytoskeletal rearrangement and inhibits the invasive capacity of gastric cancer cells
}

\author{
JIN-MING ZHU ${ }^{1 *}$ and PEI-WU YU ${ }^{2 *}$ \\ ${ }^{1}$ Department of General Surgery, Jinan Military General Hospital, Jinan, Shandong 250031; \\ ${ }^{2}$ Department of General Surgery, Southwest Hospital, Third Military Medical University, Chongqing 400038, P.R. China
}

Received January 8, 2013; Accepted May 16, 2013

DOI: $10.3892 / \mathrm{mmr} .2013 .1513$

\begin{abstract}
T-cell lymphoma invasion and metastasis-inducing factor 1 (Tiam-1) is an important member of the diffuse B-cell lymphoma (Dbl) oncogene family. In a previous study, the overexpression of Tiam-1 protein was identified by immunohistochemistry in human gastric cancer tissues, indicating that Tiam-1 may represent a candidate biomarker of the invasive and metastatic capacity of gastric cancer and for patient prognosis. In the present study, in vitro adhesion selection was used to separate two subpopulations with high (MH) or low (ML) invasive and metastatic potential from the MKN-45 human gastric cancer cell line (M0). A positive correlation was observed between Tiam-l mRNA and protein expression levels and the invasive capacity of the cells using RT-PCR and quantitative cellular-ELISA, respectively. To determine the mechanism by which Tiam-1 affects the invasive capacities of gastric cancer cells, Tiam-1 expression was downregulated in the MH subclone by liposomal transfection of antisense oligodeoxynucleotides (ASODNs). Following $48 \mathrm{~h}$ of treatment with ASODNs $(0.43 \mu \mathrm{M})$, Tiam-1 mRNA transcription and protein expression levels in $\mathrm{MH}$ cells was decreased by 80 and 24\%, respectively, compared with untreated controls. In addition, the in vitro invasive potential of MH cells was suppressed by $60 \%$. Morphological and ultrastructural observations also demonstrated that ASODN-treated MH cells exhibited a smooth surface with markedly reduced filopodia and microspikes, which resembled M0 and ML cells. In addition, cytoskeletal distribution was markedly altered from disordered to regular with reduced long filament-like structures, projections, pseudopodia on the cell surface and decreased actin bodies in the
\end{abstract}

Correspondence to: Dr Jin-Ming Zhu, Department of General Surgery, Jinan Military General Hospital, 25 Shifan Road, Jinan, Shandong 250031, P.R. China

E-mail: qingfengyunhe@sina.com

*Contributed equally

Key words: T-cell lymphoma invasion and metastasis-inducing factor 1, gastric cancer, cytoskeleton, invasion cytoplasm. Results of the current study indicate that the overexpression of Tiam-1 contributes to the invasive phenotypes of gastric cancer cells. These observations are likely to provide an improved insight into the biological mechanisms of Tiam-1 and promote the development of novel treatment strategies in gastric cancer.

\section{Introduction}

Despite recent advances in surgical treatment, chemotherapy and radiotherapy, gastric cancer remains a major global health burden. The most recent estimates found that gastric cancer is the fourth most common cancer and the second most common cause of cancer mortality worldwide $(1,2)$. The majority of gastric cancer mortalities are a result of complications associated with recurrence and metastasis, even following curative resection. However, the invasion and metastasis of gastric cancer is a complex multistep process, in which an invasive capacity is required for gastric cancer cells to undergo metastasis. The aberrant expression of specific genes is known to contribute to this transformation (3).

T-cell lymphoma invasion and metastasis inducing factor 1 (Tiam-1), an important member of the Dbl oncogene family, was identified in T-lymphoma cells using proviral tagging in combination with in vitro selection for invasiveness (4). The human Tiam-1 gene maps to the syntenic region (q22) on human chromosome 21 (21q22) and encodes a $170 \mathrm{kDa}$ transmembrane glycoprotein with intrinsic Rho GTPase activity. The gene contains a Dbl homologous (DH) domain adjacent to a pleckstrin homologous domain, a typical structure of guanine nucleotide exchange factors (GEFs) (5). As a specific GEF for Ras-related C3 botulinum toxin substrate 1 (Rac-1), a member of the Rho oncogene family, Tiam-l catalyzes the transition of Rac-l from an inactive GDP-bound state to an active GTP-bound state. In its active GTP-bound state, Rac-1 activates downstream signaling pathways associated with a number of important cellular events, including cytoskeletal reorganization, cell adhesion and migration (6). In addition, Tiam-1 has been demonstrated to increase the invasion of T-lymphoma cells, stimulate the cellular migration of fibroblasts and promote the motility of specific neurocytes. An increasing number of studies are focusing on Tiam-1 
regulatory mechanisms, as well as its role in tumor progression and metastasis. Increased Tiam-1 expression has been revealed to correlate with breast cancer grade in humans and the metastatic potential of human breast cancer cell lines in nude mice (7), moreover Tiam1 may represent a marker of progression and metastasis of hepatocellular carcinomas and colon tumors (8-10). In our previous study, negative staining for Tiam-1 protein was observed in paracarcinoma gastric mucosa, obtained from patients with gastric cancer. By contrast, another study reported that positive staining for Tiam-1 protein was detected in gastric cancer tissues (11). In addition, as the degree of histological differentiation of gastric cancer decreased, the depth of invasion was found to increase, TNM stage was upgraded and lymph node metastasis was noted. Staining corresponding to Tiam-1 protein levels in gastric cancer tissues was observed to increase, indicating that Tiam-1 may represent a candidate biomarker of the invasive and metastatic state of gastric cancer and for patient prognosis; however, the intrinsic biological mechanism via which Tiam-1 exerts it effects remains unclear.

The aim of the present study was to further define the mechanism of gastric cancer cell invasion induced by Tiam-1. Firstly, two subclones with high (MH) or low (ML) invasive and metastatic potentials were isolated from the MKN-45 human gastric cancer cell line (M0) by in vitro adhesion selection, as described previously (12). Tiam-1 expression levels and cellular morphology were compared between the subclones. In addition, the correlation between Tiam-1 expression and the in vitro invasive potential of the three cell lines was analyzed. Next, the effects of Tiam-1 downregulation on changes in cytoskeletal structure and the invasiveness of $\mathrm{MH}$ cells were investigated by in vitro transfection of antisense oligodeoxynucleotides (ASODNs). Results of the present study are likely to provide an improved insight into the mechanism of gastric cancer cell invasion and metastasis induced by Tiam-1 and promote the development of novel treatment strategies in gastric cancer.

\section{Materials and methods}

Cell culture and adhesion selection. The parental cell line MKN-45 (M0) is a poorly differentiated gastric adenocarcinoma cell line (13) and was acquired from Shanghai Institute of Digestive Disease (Shanghai Second Medical University Renji Hospital, Shanghai, China). Frozen cell stocks were thawed and grown in RPMI-1640 medium (Hyclone Laboratories Inc., Logan, UT, USA) supplemented with $10 \%$ fetal calf serum (Gibco-BRL, Carlsbad, CA, USA), L-glutamine, sodium bicarbonate, essential amino acids, antibiotics $(100 \mathrm{U} / \mathrm{ml}$ penicillin and $100 \mu \mathrm{g} / \mathrm{ml}$ streptomycin) and sodium pyruvate. The medium was replaced with fresh medium every 2-3 days to maintain optimal growth at $37^{\circ} \mathrm{C}$ in a humidified atmosphere containing $5 \% \mathrm{CO}_{2}$. At $\sim 80 \%$ confluence, cells were passaged at a ratio of $1: 3$ and detached using a $0.25 \%(\mathrm{w} / \mathrm{v})$ trypsin-EDTA solution every 5-6 days.

M0 cells were selected for adhesion on mouse laminin-1-coated substrates (Sigma-Aldrich, St Louis, MO, USA), as described previously (12). Briefly, culture dishes (diameter, $35 \mathrm{~mm}$ ) were coated with $50 \mu \mathrm{g}$ laminin-1 in phosphate buffered saline (PBS) and air-dried overnight at $4^{\circ} \mathrm{C}$.
Subconfluent M0 cells were incubated in $0.25 \%$ trypsin-EDTA solution, washed and resuspended in serum-free medium. The cell suspension $\left(2.5 \times 10^{5}\right.$ cells/dish) was incubated with laminin-1 coated substrate for $1 \mathrm{~h}$ and attached and unattached cells were collected separately. The attached cells were cultured to subconfluence on laminin-1 coated dishes, then selected again for adhesion. Unaffected cells were prepared following the same protocol. Serial adhesion selection was performed 20 times, then attached $(\mathrm{MH})$ or unattached (ML) cells were maintained under normal culture conditions. Two passages after the last adhesion selection, the selected cells (MH and ML) were used for further experiments.

Antisense treatment of MH cell subclones. The design of one 18-mer ASODN was based on the nucleotide sequence of human Tiam-1, targeted to the DH domain, while sense oligonucleotide (SODN) was used as a control. Specific primers against ASODN and SODN were derived from previously published sequences (14) and determined using a BLAST search of a current EMBL database/NCBI-Genebank database (http://blast.ncbi.nlm.nih.gov/). The sequences used were as follows: SODN, 5'-* G*AT CTG CGA GCT CCT GG ${ }^{*} \mathrm{~A}^{*}-3$ '; and ASODN, 5'-*T*C AGG AGC TCG CAG AT C $^{*}-3$ ' ('represents phosphorothioate linkages). All ODNs were synthesized using the Applied Biosystems 391 DNA synthesizer (Perkin Elmer Applied Biosystems, Inc., Foster City, CA, USA).

$\mathrm{MH}$ cells in log-phase growth $\left(2.5 \times 10^{5}\right.$ cells $)$ were plated onto culture dishes (diameter, $35 \mathrm{~mm}$ ) and incubated under standard culture conditions for $24 \mathrm{~h}$, then transfected with an ASODN or SODN of Tiam-1 using DOTAP liposomal transfection reagent (Roche $\mathrm{GmbH}$, Mannheim, Germany). The preparation of liposome-ODN was performing according to the manufacturer's instructions. Prior to treatment, ODNs and liposomes (ratio w/v, 5/15 $\mu \mathrm{l} ; 1: 3$ ) were dissolved separately in HEPES-buffered saline solution (20 mm HEPES, $150 \mathrm{~mm}$ $\mathrm{NaCl}$; $\mathrm{pH} 7.4$ ) and mixed at room temperature for $15 \mathrm{~min}$. The optimal working conditions for lipofectin-ODN were determined previously in preliminary experiments (data not shown). Experimental cells were treated with liposome-ASODN at a final concentration of $0.43 \mu \mathrm{M}$. Control treatment groups were serum-free culture medium only, liposome only (15 $\mu \mathrm{l} / \mathrm{dish})$ and liposome-SODN $(0.43 \mu \mathrm{M})$. The culture medium was removed and cells were exposed to lipofection solution for $8 \mathrm{~h}$. At the end of the incubation with ODN-liposome, the medium was replaced with fresh serum-containing culture medium ( $3 \mathrm{ml} /$ dish) and placed into an incubator. Analysis of Tiam-1 mRNA expression in $\mathrm{MH}$ cells was performed $48 \mathrm{~h}$ following transfection.

Reverse transcription polymerase chain reaction (RT-PCR). Total RNA was extracted using TriPure Isolation Reagent (Boehringer Mannheim, Mannheim, Germany) according to the manufacturer's instructions, and its content and purity were evaluated by ultra-violet spectrophotography. cDNA was synthesized in $20-\mu \mathrm{l}$ reaction volumes containing $1 \mu \mathrm{g}$ total RNA, $0.2 \mu \mathrm{g} / \mu 1$ random hexamer primer, $4 \mu 15 \mathrm{X}$ reaction buffer, $20 \mathrm{U} / \mu \mathrm{l}$ ribonuclease inhibitor, $10 \mathrm{mmol} / \mathrm{l}$ deoxyribonucleotide triphosphate (dNTPs) and 20 units Moloney murine leukemia virus reverse transcriptase (G-Biosciences \& Geno Technology Co., St. Louis, USA). Procedures were performed 
according to the manufacturer's instructions of the First Strand cDNA synthesis kit (Sangon Biological Engineering Co., Shanghai, China).

For the semi-quantitative assay, a standard calibration curve was created to determine the optimum number of cycles (15-35 cycles); the most appropriate number of cycles was 30 (data not shown). PCR was performed using the following materials: $2 \mu \mathrm{l} \mathrm{cDNA}, 5 \mu \mathrm{l} 10 \mathrm{X}$ buffer, $4 \mu \mathrm{l} \mathrm{MgCl}_{2}$ ( $25 \mathrm{mmol} / \mathrm{l}), 8 \mu \mathrm{ldNTPs}(2.5 \mathrm{mmol} / \mathrm{l}), 0.5 \mu \mathrm{l}$ of each forward and reverse amplification primer $(25 \mu \mathrm{mol} / \mathrm{l})$ and $0.5 \mu \mathrm{l} \mathrm{Ex} \mathrm{Taq}$ DNA polymerase (2.5 units; Takara Biotechnology Co., Ltd., Dalian, China) in a $50 \mu 1$ reaction volume. Reactions were performed using the PTC-100TM PCR system (MJ Research Inc., Watertown, MA, USA) under the following conditions: 30 cycles of $94^{\circ} \mathrm{C}(45 \mathrm{sec}), 54^{\circ} \mathrm{C}(1 \mathrm{~min})$ and $72^{\circ} \mathrm{C}(1.5 \mathrm{~min})$. The following primer pairs (synthesized by Sangon Biological Engineering Co.) were used to amplify fragments of Tiam-1 (818 bp) and GADPH (310 bp) cDNAs: Tiam-1, forward 5'-TTC TCA CCA GTC TGT TCA GC-3' and reverse 5'-CCA GAC TTG GAA TCC TCA GA-3'; and GADPH, forward 5'-AGG TCC ACC ACT GAC ACG TT-3' and reverse 5'-GCC TCA AGA TCA TCA GCA AT-3'.

PCR products $(5 \mu \mathrm{l})$ amplified from the Tiam- 1 or GADPH genes were mixed and underwent $2 \%$ ethidium bromide agarose gel electrophoresis with molecular weight standards. The band intensities for Tiam-1 and GADPH were estimated using a Bio-Rad gel documentation 2000 system (Bio-Rad, Hercules, CA, USA). The intensity of Tiam-1 gene expression were reported as the ratio $\left(R_{V}\right)$ of Tiam-1 to GADPH (as an internal control). Specimens were analyzed three times and values were averaged.

Quantitative cell-ELISA. MH cells ( $1 \times 10^{5}$ cells) in log-phase growth were plated onto 96-well plates, incubated under standard culture conditions for $24 \mathrm{~h}$ and transfected as described. Control groups were prepared following the same protocol. Quantitative cellular-ELISA of the Tiam-1 protein in gastric cancer cells was performed following transfection for $48 \mathrm{~h}$, as described previously (15).

In brief, culture medium was removed from the 96-well plates and the cells were washed twice with PBS, fixed with $4 \%$ paraformaldehyde (w/v in PBS) for $1 \mathrm{~h}$ and removed. Following three PBS washes, cells were blocked with $10 \%$ (w/v) goat serum albumin in PBS at $4^{\circ} \mathrm{C}$ for $15 \mathrm{~min}$ and then incubated at $37^{\circ} \mathrm{C}$ for $2 \mathrm{~h}$ with a 1:100 working dilution of rabbit polyclonal antibody (50 $\mu \mathrm{l} /$ well) against human Tiam-1 (Santa Cruz Biotechnology, Inc., Santa Cruz, CA, USA). Next, cells were washed 3 times with $0.05 \%$ Tween-20/PBS and incubated at $37^{\circ} \mathrm{C}$ for $1 \mathrm{~h}$ with a $1: 5,000(50 \mu \mathrm{l} /$ well $)$ working dilution of horseradish peroxidase-conjugated goat anti-rabbit secondary antibody (Zhongshan Biotechnology Co., Ltd., Beijing, China). Following four washes with $0.05 \%$ Tween-20/PBS, equal amounts of A and B fluid (50 $\mu \mathrm{l}$ each) containing 3,3',5,5'-tetramethylbenzidine peroxidase chromogenic reagent (KPL, Inc., Gaithersburg, MD, USA) were added to react in the dark for $10 \mathrm{~min}$. The reaction was terminated with $50 \mu \mathrm{l} /$ well $\mathrm{HCl}(1 \mathrm{~mol} / \mathrm{l})$ under oscillation for $30 \mathrm{sec}$ and the absorbance at $450 \mathrm{~nm}$ was measured using an ELISA plate reader (Sunrise; Tecan Group, Ltd., Männedorf, Switzerland). Wells were washed with distilled water, followed by the addi- tion of $0.08 \%(\mathrm{w} / \mathrm{v})$ crystal violet $(100 \mu \mathrm{l} /$ well $)$ and incubation at room temperature for $20 \mathrm{~min}$. The wells were then washed again with distilled water and the reaction was terminated using $33 \%(\mathrm{w} / \mathrm{v})$ glacial acetic acid (100 $\mu \mathrm{l} /$ well) at room temperature for $30 \mathrm{~min}$ and the absorbance was measured at $550 \mathrm{~nm}$. The intensity of Tiam-1 protein was reported as the ratio $\left(\mathrm{R}_{\mathrm{D}}\right)$ of D450 to D550. Specimens were analyzed three times and the values were averaged. Negative controls consisted of omission of the primary antibody.

Morphological and ultrastructural observations. To examine morphological changes, cells were directly cultured on slides and washed twice with PBS. Next, the cells were stained with hematoxylin and eosin (H\&E) and observed under a light microscope, and representative images were captured.

For scanning electron microscopy (SEM), cells were grown on cover slips and fixed with $2.5 \%$ (w/v) glutaraldehyde at $4^{\circ} \mathrm{C}$ overnight. Following this, cells were washed with PBS, treated with $1 \%(\mathrm{w} / \mathrm{v})$ osmium tetroxide (TAAB Laboratories Equipment Ltd., Berkshire, UK) at $4^{\circ} \mathrm{C}$ for $1 \mathrm{~h}$, washed three times with PBS and dehydrated using a gradient ethanol. SEM specimens were dried using a critical point dryer (Hcp-2; Hitachi Ltd., Tokyo, Japan) and the sputter was coated with $\mathrm{Pt}+\mathrm{Cd}$ using an ion sputtering device (Oie-102; Hitachi Ltd.). Cell surface alteration was observed under an SEM microscope (AMRay 1000B, Bedford, MA, USA).

Cytoskeletal staining. Cytoskeletal staining was performed as described previously (16). Following two washes with PBS, cells cultured on slides were permeabilized with $0.1 \%$ Triton-X-100 3 times for $8 \mathrm{~min}$, washed in M-buffered solution (50 $\mathrm{mM}$ imidazole, $50 \mathrm{mM}$ potassium chloride, $0.5 \mathrm{mM}$ magnesium chloride, 2 mM EGTA, $0.1 \mathrm{mM}$ EDTA, $1 \mathrm{mM}$ mercaptoethanol, $4 \mathrm{M}$ glycerol and distilled water to $1,000 \mathrm{ml}) 3$ times for $4 \mathrm{~min}$ and then fixed with $3 \%(\mathrm{w} / \mathrm{v})$ glutaraldehyde at room temperature for $10 \mathrm{~min}$. Next, cells were washed in M-buffered solution 3 times for $3 \mathrm{~min}$ and stained with coomasssie brilliant blue solution (0.2 g R-250, $46.5 \mathrm{ml}$ methanol, $7 \mathrm{ml}$ glacial acetic acid and $46.5 \mathrm{ml}$ distilled water) at room temperature for $40 \mathrm{~min}$, followed by PBS washing, air-drying, dimethyl benzene clearing and neutral gum sealing. Cells were then viewed under a light microscope (LH50A; Olympus Optical Co., Tokyo, Japan).

In vitro invasion assay. In vitro cell invasion was analyzed using 24-transwell units (Millipore, Billerica, MA, USA), as described previously (17). Polyvinylpyrrolidone-free polycarbonate filters (diameter, $12 \mu \mathrm{m}$; pore size, $8 \mu \mathrm{m}$; Millipore) were coated with $30 \mu \mathrm{g}$ Matrigel/filter (BD Biosciences, Franklin Lakes, NJ, USA). NIH-3T3 cell-conditioned media was used as a chemoattractant and was obtained by culturing cells for $24 \mathrm{~h}$ in serum-free RPMI-1640 medium. Cells in serum-free RPMI-1640 medium ( $400 \mu$ l containing $1 \times 10^{5}$ cells) were added to the upper compartment of the chamber, while $600 \mu \mathrm{l}$ conditioned medium was added to the lower compartment. Following $72 \mathrm{~h}$ incubation at $37^{\circ} \mathrm{C}$ in humidified $95 \%$ air with $5 \% \mathrm{CO}_{2}$, basement membrane Matrigel and cells on the upper side of the filter were removed by wiping with a cotton swab. Cells that had migrated to the lower surface of the filters were fixed and stained with $H \& E$. The invasive potential of cells was determined by measuring the number of 
Table I. In vitro invasive potential of gastric cancer cells.

\begin{tabular}{lcccr}
\hline & \multicolumn{4}{c}{ Cells (n) } \\
\cline { 2 - 4 } Variable & M0 & ML & MH & \multirow{2}{*}{ F-value } \\
\hline Cells/field & $11.67 \pm 3.79$ & $9.67 \pm 3.06$ & $24.33 \pm 8.02^{\mathrm{a}, \mathrm{b}}$ & 6.470
\end{tabular}

Data are presented as the mean $\pm \mathrm{SD} .{ }^{\mathrm{a}} \mathrm{P}<0.05 \mathrm{vs} . \mathrm{M} 0 ;{ }^{\mathrm{b}} \mathrm{P}<0.05 \mathrm{vs}$. ML. MO, MKN-45 human gastric cancer cell line; MH, cell subpopulation with a high invasive and metastatic potential; ML, cell subpopulation with a low invasive and metastatic potential.

Table II. Tiam-1 mRNA and protein expression levels in gastric cancer cells.

\begin{tabular}{lcccr}
\hline & \multicolumn{4}{c}{ Cells } \\
\cline { 2 - 4 } Ratio & M0 & ML & MH & \multirow{2}{*}{ F-value } \\
\hline $\mathrm{R}_{\mathrm{V}}$ & $0.759 \pm 0.047$ & $0.743 \pm 0.039$ & $0.855 \pm 0.051^{\mathrm{a}, \mathrm{b}}$ & 5.269 \\
$\mathrm{R}_{\mathrm{D}}$ & $0.911 \pm 0.104$ & $0.892 \pm 0.101$ & $1.262 \pm 0.165^{\mathrm{a}, \mathrm{b}}$ & 13.429
\end{tabular}

Data are presented as the mean $\pm \mathrm{SD} .{ }^{\mathrm{a}} \mathrm{P}<0.05$ vs. $\mathrm{M} 0$; ${ }^{\mathrm{b}} \mathrm{P}<0.05$ vs. ML. Tiam-1, T-cell lymphoma invasion and metastasis-inducing factor $1 ; \mathrm{R}_{\mathrm{D}}$, ratio of $\mathrm{D} 450$ to $\mathrm{D} 550 ; \mathrm{R}_{\mathrm{V}}$, ratio of Tiam-1 to GADPH; $\mathrm{MO}, \mathrm{MKN}-45$ human gastric cancer cell line; $\mathrm{MH}$, cell subpopulation with a high invasive and metastatic potential; ML, cell subpopulation with a low invasive and metastatic potential.

cells that migrated to the lower side of the filters through the basement membrane matrigel and pores. The number of cells that penetrated the filter was counted in 10 microscopic fields of each filter. Assays were performed in triplicate.

Statistical analysis. Quantitative data are presented as the mean \pm SD. All data were analyzed statistically by one-way ANOVA followed by a least significant difference test, using the SPSS 10.0 software program (SPSS Inc., Chicago, IL, USA). $\mathrm{P}<0.05$ was considered to indicate a statistically significant difference. Correlations were calculated using the Spearman correlation coefficient.

\section{Results}

In vitro invasive potential of $M O, M L$ and $M H$ cells. $\mathrm{MH}$ and ML cells, which exhibit different invasive and metastatic potentials, were subcloned from the MKN-45 human gastric cancer cell line (M0) by adhesion selection. A transwell assay was performed to compare the invasive capacity of the three cell strains in vitro. As demonstrated in Table I and Fig. 1, MH cells exhibited a higher invasive potential than M0 and ML cells in vitro. This is consistent with results of a previous study on tumor-cell transplantation and experimental metastasis in nude mice (12).

Tiam-1 mRNA and protein expression levels in MO, ML and MH cells. Since Tiam-l has been previously reported to be

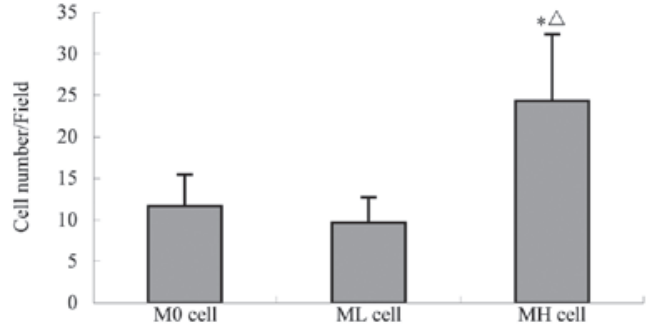

Figure 1. In vitro invasive potential of $\mathrm{M} 0, \mathrm{ML}$ and $\mathrm{MH}$ cells. ${ }^{*} \mathrm{P}<0.05$ vs. $\mathrm{M} 0$ ${ }^{\triangle} \mathrm{P}<0.05$ vs. ML. MO, MKN-45 human gastric cancer cell line; $\mathrm{MH}$, cell subpopulation with a high invasive and metastatic potential; ML, cell subpopulation with a low invasive and metastatic potential.

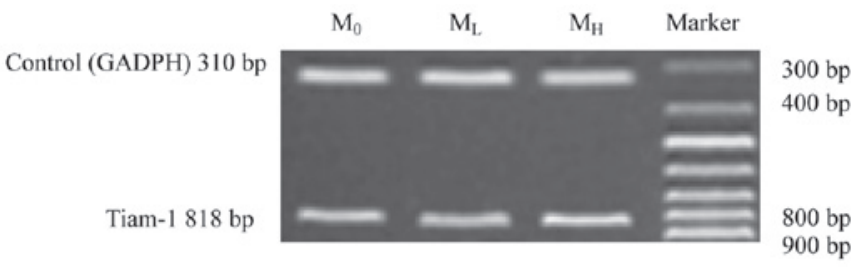

Figure 2. RT-PCR analysis demonstrated that the MH cell subclone expressed Tiam-1 as a marked 818 bp-band, while M0 and ML cells expressed faint bands. RT-PCR, reverse transcription polymerase chain reaction; MO, MKN-45 human gastric cancer cell line; $\mathrm{MH}$, cell subpopulation with a high invasive and metastatic potential; ML, cell subpopulation with a low invasive and metastatic potential; Tiam-1, T-cell lymphoma invasion and metastasis-inducing factor 1 .

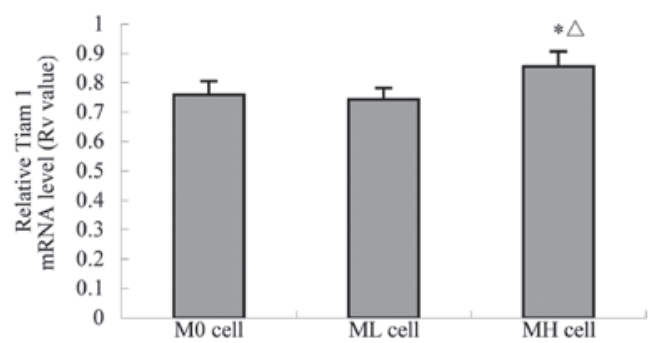

Figure 3. Tiam-1 mRNA expression levels in M0, ML and MH cells ${ }^{*} \mathrm{P}<0.05$ vs. $\mathrm{M} 0 ;{ }^{\triangle} \mathrm{P}<0.05$ vs. ML. Tiam-1, T-cell lymphoma invasion and metastasis-inducing factor 1 ; MO, MKN-45 human gastric cancer cell line; $\mathrm{MH}$, cell subpopulation with a high invasive and metastatic potential; ML, cell subpopulation with a low invasive and metastatic potential; $\mathrm{RV}$, ratio of Tiam-1 to GADPH.

associated with tumor invasion and metastasis, Tiam-1 mRNA and protein expression levels in M0, ML and MH cells were analyzed by RT-PCR and quantitative cellular-ELISA. The expression of Tiam-1 mRNA and protein in the three strains was positive; however, levels in MH cells were observed to be markedly higher than those of M0 and ML cells (Table II; Figs. 2-4)

These observations were analyzed further, revealing a positive correlation between the expression levels of Tiam-l mRNA or protein and the invasive capacity of the cells (Spearman correlation coefficient, $r=1.000 ; \mathrm{P}<0.01$ ).

Antisense treatment downregulates the expression of Tiam-1 mRNA and protein in MH cells. To confirm the effect of Tiam-1 ASODN on MH cells, RT-PCR and quantitative 
Table III. Tiam-1 mRNA and protein expression levels in gastric cancer cells.

\begin{tabular}{lcccr}
\hline Ratio & Control & Liposome & SODN-liposome & ASODN-liposome \\
\hline $\mathrm{R}_{\mathrm{V}}$ & $0.801 \pm 0.065^{\mathrm{a}}$ & $0.789 \pm 0.054^{\mathrm{a}}$ & $0.754 \pm 0.039^{\mathrm{a}}$ & $0.162 \pm 0.018$ \\
$\mathrm{R}_{\mathrm{D}}$ & $1.290 \pm 0.182^{\mathrm{a}}$ & $1.237 \pm 0.108^{\mathrm{a}}$ & $1.234 \pm 0.103^{\mathrm{a}}$ & $0.982 \pm 0.119$ \\
\hline
\end{tabular}

Data are presented as the mean $\pm \mathrm{SD}$. ${ }^{\mathrm{P}}<0.05$ vs. ASODN-liposome-treated MH cells. Tiam-1, T-cell lymphoma invasion and metastasis-inducing factor 1; $\mathrm{R}_{\mathrm{D}}$, ratio of D450 to D550; $\mathrm{R}_{\mathrm{V}}$, ratio of Tiam-1 to GADPH; SODN, sense oligonucleotide; ASODN, antisense oligodeoxynucleotide; $\mathrm{MH}$, cell subpopulation with a high invasive and metastatic potential.

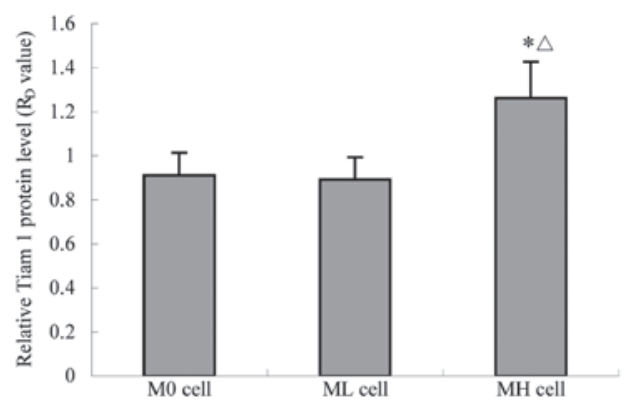

Figure 4. Tiam-1 protein expression levels among M0, ML and MH cells. ${ }^{*} \mathrm{P}<0.05$ vs. M0; ${ }^{\circ} \mathrm{P}<0.05$ vs. ML. Tiam-1, T-cell lymphoma invasion and metastasis-inducing factor 1 ; MO, MKN-45 human gastric cancer cell line; $\mathrm{MH}$, cell subpopulation with a high invasive and metastatic potential; ML, cell subpopulation with a low invasive and metastatic potential; RD, ratio of D450 to D550.

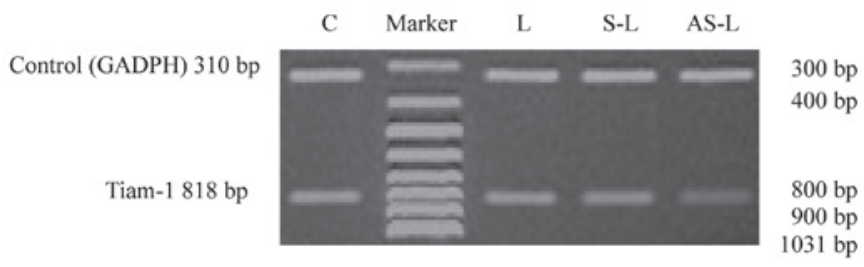

Figure 5. RT-PCR analysis revealed that ASODN-liposome (AS-L)-treated MH cells faintly expressed Tiam-1 as an 818-bp band. Untreated (control, C), liposome only-treated (L) and SODN-liposome (S-L)-treated MH cells expressed strong bands. RT-PCR, reverse transcription polymerase chain reaction; SODN, sense oligonucleotide; ASODN, antisense oligodeoxynucleotide; Tiam-1, T-cell lymphoma invasion and metastasis-inducing factor 1; $\mathrm{MH}$, cell subpopulation with a high invasive and metastatic potential.

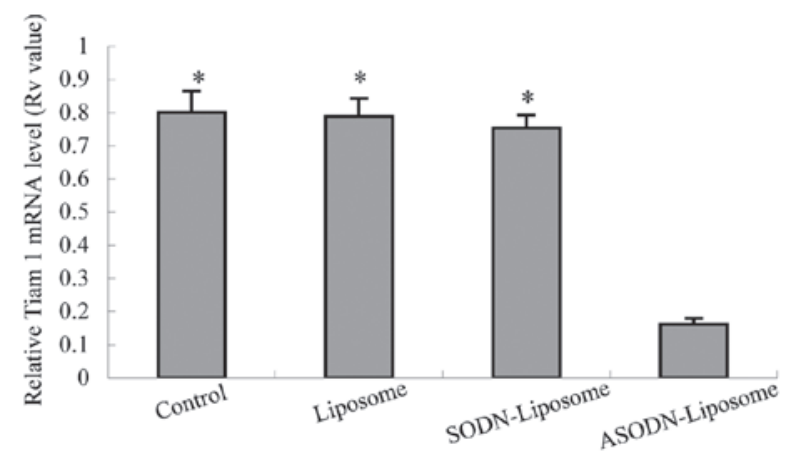

Figure 6. Tiam-1 mRNA expression levels following antisense treatment. ${ }^{*} \mathrm{P}<0.05$ vs. ASODN-liposome-treated MH cells. Tiam-1, T-cell lymphoma invasion and metastasis-inducing factor 1; SODN, sense oligonucleotide; ASODN, antisense oligodeoxynucleotide; $\mathrm{MH}$, cell subpopulation with a high invasive and metastatic potential; RV, ratio of Tiam-1 to GADPH.

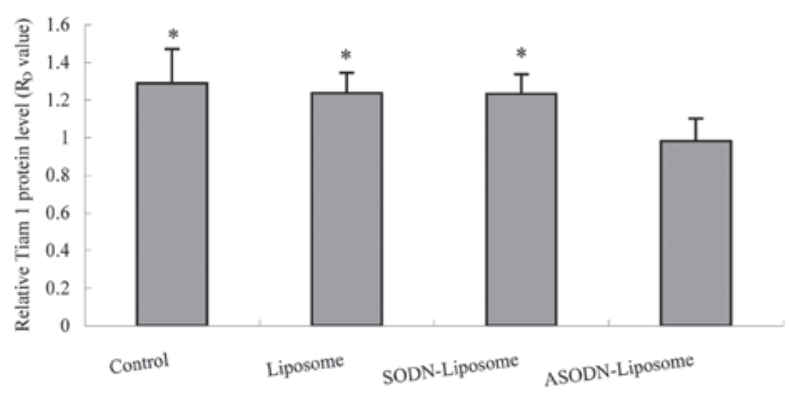

Figure 7. Tiam-1 protein expression levels following antisense treatment. $\mathrm{P}<0.05$, vs. ASODN-liposome treated MH cells. Tiam-1, T-cell lymphoma invasion and metastasis-inducing factor 1 ; SODN, sense oligonucleotide; ASODN, antisense oligodeoxynucleotide; $\mathrm{MH}$, cell subpopulation with a high invasive and metastatic potential; RD, ratio of D450 to D550.

cell-ELISA were performed. The optimum conditions for antisense treatment, determined in preliminary experiments (data not shown), were $0.43 \mu \mathrm{M}$ ODN, ratio (w/v) of ODN/liposome $=1: 3,4 \mathrm{~h}$ exposure and $48 \mathrm{~h}$ recovery. Following ASODN-liposome treatment, the expression of Tiam-1 in MH cells was significantly inhibited compared with untreated (control), liposome- or SODN-liposome-treated samples (Table III). RT-PCR revealed a faint Tiam-1 band in $\mathrm{MH}$ cells following ASODN treatment. By contrast, the band was significantly stronger in the other three groups. No significant difference between the intensities of the stronger bands was identified (Figs. 5 and 6). Quantitative cell-ELISA of MH cells transfected with ASODN-liposome also revealed a significant reduction in Tiam-1 protein levels compared with untreated, liposome- or SODN-liposome treated groups (Fig. 7).

Alterations in cellular morphology and ultrastructure following antisense treatment. Following H\&E staining, cells were observed under a light microscope. M0 and ML cells were floating and exhibited round epithelial-like morphologies with a large nucleocytoplasmic ratio; no differences in morphology were found between M0 and MH cells. However, $\mathrm{MH}$ cells were observed to be multiform compared with the uniform appearance of M0 and ML cells. A number of MH cells were observed to exhibit a polygonal morphology with longer cytoplasmic processes (Fig. 8). Following Tiam-1 ASODN treatment, the morphology of MH cells resembled that of M0 and ML cells.

SEM revealed abundant microvilli, cytoplasmic processes and large pseudopodia dotted on the surface of MH cells. 

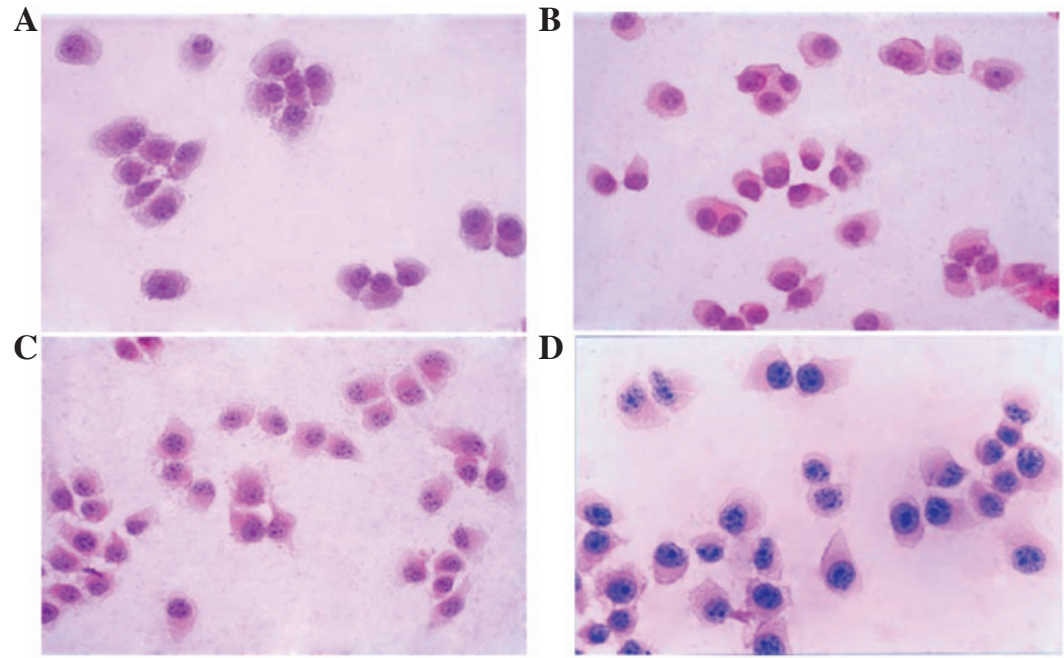

Figure 8. Gastric cancer cell morphology. Gastric cancer cells stained with H\&E were observed by light microscopy. (A) M0, (B) ML, (C) MH and (D) antisense-treated MH cells (magnification, x200). H\&E, hematoxylin and eosin; MO, MKN-45 human gastric cancer cell line; MH, cell subpopulation with a high invasive and metastatic potential; ML, cell subpopulation with a low invasive and metastatic potential.
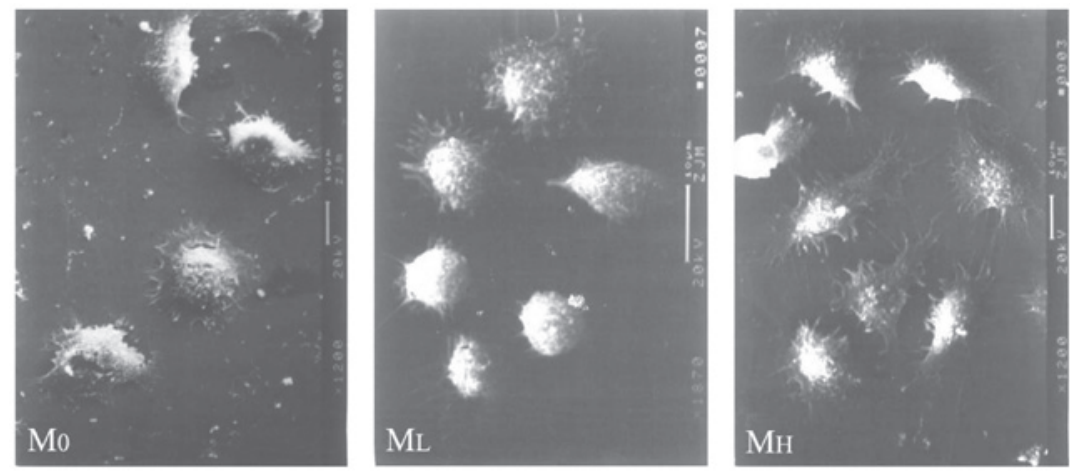

Figure 9. Scanning electron microscopy. Ultrastructural features of gastric cancer cells. MO, MKN-45 human gastric cancer cell line; MH, cell subpopulation with a high invasive and metastatic potential; ML, cell subpopulation with a low invasive and metastatic potential. Magnification, x1,200.
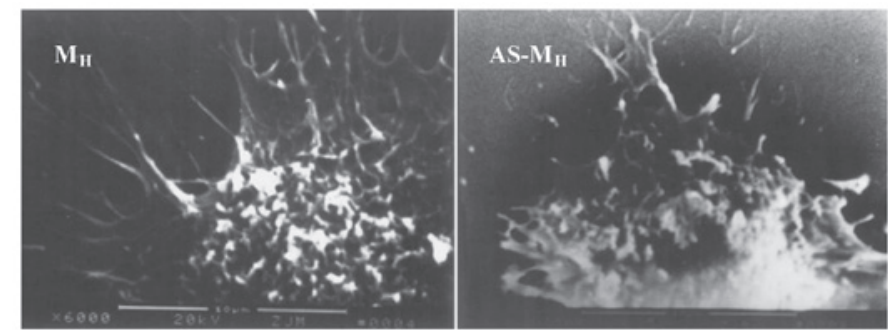

Figure 10. Scanning electron microscopy. Ultrastructural alterations of MH cells following antisense treatment. MH, cell subpopulation with a high invasive and metastatic potential; AS-MH, antisense treatment MH. Magnification, x6,000.
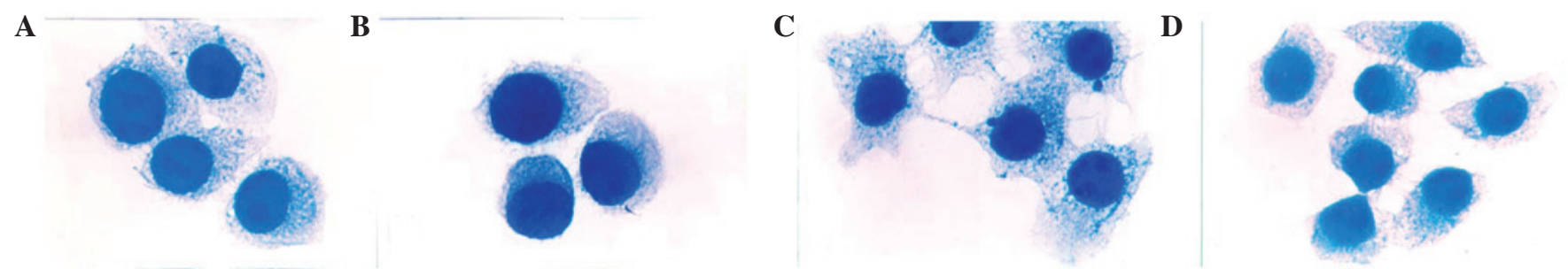

Figure 11. Coomasssie brilliant blue staining of cytoskeletal changes in (A) M0, (B) ML, (C) MH and (D) ASODN-treated MH cells. Images captured under an immersion objective (magnification, ). MO, MKN-45 human gastric cancer cell line; $\mathrm{MH}$, cell subpopulation with a high invasive and metastatic potential; ML, cell subpopulation with a low invasive and metastatic potential; ASODN, antisense oligodeoxynucleotide. Magnification, x1,000. 
Table IV. Effect of antisense treatment on the in vitro invasive potential of $\mathrm{MH}$ cells.

\begin{tabular}{llcl}
\hline & \multicolumn{2}{c}{ Cells (n) } & \\
\cline { 2 - 3 } Variable & Untreated & ASODN-treated & F-value \\
\hline Cells/field & $25.67 \pm 6.11$ & $10.33 \pm 2.52^{\mathrm{a}}$ & 16.153 \\
\hline
\end{tabular}

Data are presented as the mean $\pm \mathrm{SD} .{ }^{\mathrm{a}} \mathrm{P}<0.05$ vs. ASODN-treated MH cells. ASODN, antisense oligodeoxynucleotide; MH, cell subpopulation with a high invasive and metastatic potential.

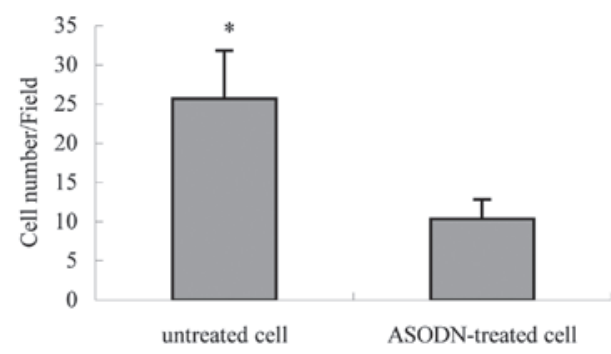

Figure 12. In vitro invasive potential of ASODN-treated or untreated $\mathrm{MH}$ cells. ${ }^{*} \mathrm{P}<0.05$ vs. ASODN-treated MH cells. ASODN, antisense oligodeoxynucleotide; $\mathrm{MH}$, cell subpopulation with a high invasive and metastatic potential.

Certain processes extended a considerable distance, radiated in all directions or formed bulges at their ends. By contrast, the processes of M0 and ML cells were short and compact. No desmosomes, tight conjunctions or other cell junction structures were observed in the three cell types. Following antisense treatment, $\mathrm{MH}$ cells exhibited a smooth surface with markedly reduced filopodia and microspikes (Figs. 9 and 10).

Cytoskeletal structure and reorganization following antisense treatment. Cells were stained with coomasssie brilliant blue R-250 and viewed under an immersion objective. The cytoskeletal structure of M0, ML and MH cells formed a complicated cytoskeletal network in the cytoplasm and was markedly more concentrated in perinuclear areas. Bundles of cytoskeletal filaments were stretched and scattered intercellularly. Compared with M0 and ML cells, cytoskeletal alignment in $\mathrm{MH}$ cells was disordered, accompanied with more dotted actin bodies, cytoplasmic processes and long filament-like structures. By contrast, no differences in cytoskeletal alignment were observed between M0 and ML cells. Following antisense treatment, cytoskeletal distribution in $\mathrm{MH}$ cells changed from irregular to regular, with reduced long filament-like structures on the cell surface and dotted actin bodies in the cytoplasm, and resembled that of M0 and ML cells (Fig. 11).

Tiam-1 ASODN treatment downregulates the invasiveness of $\mathrm{MH}$ cells in vitro. An in vitro transwell assay revealed that the number of antisense-treated $\mathrm{MH}$ cells penetrating the artificial basement membrane was $10.33 \pm 2.52$ cells/high power field, which was significantly lower than that of untreated $\mathrm{MH}$ cells $(25.67 \pm 6.11$ cells/high power field; $\mathrm{P}<0.05$; Table IV, Fig. 12).

\section{Discussion}

Tumor cell invasion and metastasis depends on a unique set of biological properties that enable malignant cells to complete all steps of the metastatic cascade. A tumor mass commonly consists of various cell subclones, a phenomenon known as tumor heterogeneity (18). Since subclones are from the same parental cell line, thus having the same genetic background, the differences in their phenotypes are likely to have a number of underlying molecular mechanisms $(19,20)$. In-depth study of the differences between subclones may provide novel insight into the mechanisms of tumor cell invasion and metastasis.

Members of the Rho GTPase family have emerged as key players in the regulation of a diverse set of biological activities, including actin organization, focal complex/adhesion assembly, cell motility, cell polarity and gene transcription, the importance of which is becoming evident in cancer progression, particularly in the area of metastasis $(21,22)$. As an upstream regulator of Rho-GTPases, particularly Rac-1, the potential role of Tiam-1 in tumor invasion and metastasis is becoming increasingly apparent.

In the present study, two subclones with a high $(\mathrm{MH})$ or low (ML) invasive and metastatic potential were acquired from the MKN-45 human gastric cancer cell line (M0) using in vitro adhesion selection on laminin-1-coated dishes (12), and Tiam-1 expression levels were compared. Results indicated that Tiam-1 mRNA and protein expression levels in $\mathrm{MH}$ cells were markedly higher than that of M0 and ML cells. In addition, a positive correlation between the expression of Tiam-1 and the in vitro invasive potentials of M0, ML and $\mathrm{MH}$ cells was observed. These results are consistent with previous observations (11) and implicate a role for Tiam-1 in the invasive process of human gastric cancer.

Cytoskeletal components, including microtubules, microfilaments and intermediate filaments, are responsible for the maintenance of cell morphology, direction of cell locomotion, cell spreading, cell migration and stability of cell-substrate contact in normal cells (23). Alterations in cytoskeletal structures, such as reduced microtubules, disruption of stress fibers and redistribution of actin-filaments in tumor cells, have been reported to be important determinants of malignancy in tumor cells, enabling cells to transform and transmigrate within tissues (24). In the present study, analysis of the cellular morphology and ultrastructure of gastric cancer cells revealed that M0 and ML cells were floating and exhibited round epithelial-like structures. By contrast, $\mathrm{MH}$ cells were multiform and a number of cells were polygonal with long cytoplasmic processes. SEM also revealed abundant microvilli, cytoplasmic processes and large microhills located on the MH cell surface. Specific processes were observed to extend considerable distances, radiate in all directions or form bulges at their ends. By contrast, M0 and ML cells were observed to exhibit short and compact processes. Following staining with coomassie brilliant blue, the cytoskeletal structure of M0, ML and MH cells was observed to form a complicated cytoskeletal network in the cytoplasm. The network was particularly concentrated in perinuclear areas and bundles of 
cytoskeletal filaments passed through the cell wall to interact with cytoskeletal arrays of adjacent cells. Compared with M0 and ML cells, cytoskeletal alignment in MH cells was disordered, accompanied with increased dotted actin bodies, cytoplasmic processes and long filament-like structures, whereas no marked differences were observed between M0 and ML cells. The differences in cytoskeletal structure may be due to cytodynamic activities associated with dissemination and implantation, based on the invasive and metastatic potentials of M0, ML and MH cells. As Tiam-1 is known to regulate the reorganization of cytoskeletal structure, differences in the morphological characteristics of gastric cancer cells with different invasive and metastatic potentials may correlate with Tiam-1 expression.

ASODNs bind and inactivate specific RNA sequences and represent one of the best tools for studying gene function, the regulation of gene expression and interactions between gene products. Intracellular delivery of ASODNs results in the downregulation of target gene expression by inhibiting transcription or translation without affecting other cellular functions. To validate the role of Tiam-1 in gastric cancer cell invasion, a 18-mer ASODN was used to block Tiam-1 expression in $\mathrm{MH}$ cells and the subsequent changes in cellular morphology and the in vitro invasiveness of $\mathrm{MH}$ cells were analyzed. As unmodified phosphodiester oligomers are susceptible to nucleases, partial phosphorothioate oligomers were used, which are relatively nuclease-resistant. Results demonstrated that the treatment of $\mathrm{MH}$ cells with ASODNs $(0.43 \mu \mathrm{M}, 48 \mathrm{~h})$ decreased Tiam-1 mRNA transcription and protein expression by 70 [(0.801-0.162)/0.801] and $65 \%$ [(1.290-0.982)/1.290] respectively, compared with untreated controls. In addition, the in vitro invasive potential of ASODN-treated MH cells was suppressed by $75 \%$ [(25.67-10.33)/25.67]; however, cells treated with SODN or lipofectin alone were not affected. Morphological and ultrastructural observations also revealed that ASODN-treated MH cells exhibited a smooth surface with markedly reduced filopodia and microspikes, which resembled M0 and ML cells. Cytoskeletal distribution in ASODN-treated MH cells was markedly altered from disordered to regular, with reduced long filament-like structures, projections, pseudopodia at the cell surface and reduced actin bodies in the cytoplasm. Our findings showed that Tiam-1 induces gastric cancer cell invasion by regulating cytoskeletal reorganization, which caused the depolymerization of microtubules and microfilaments, leading to disordered, actively motile properties, cell detachment and a metastatic potential in gastric cancer cells. Previous observations showed that Tiam-1 contributed to the cytoskeletal reorganization required during cell migration and neurite extension by activation of Rac 1 (25). Whether this is the case in gastric cancer cell invasion it is not yet known.

In addition, abnormalities in the expression and functional activity of cell adhesion molecules are implicated in the invasive and metastatic progression of tumor cells. Intercellular and cell-matrix adhesion molecules have been shown to have roles in addition to acting as cementing substances, such as regulating cell polarity, differentiation, invasion and migration (26). A number of these cellular events are mediated through direct associations with the cytoskeletal network and interactions between the cytoskeletal network. Therefore, the manipulation of molecules associated with the cytoskeleton requires further investigation (27). The in vitro cell model of laminin-1 adhesion selection used in the present study represents a simple and time-effective method to screen a specific phenotypic cell subpopulation and is likely to be useful for future analysis of the cytoskeleton.

Laminin-1, a major basement membrane glycoprotein, promotes the malignant phenotype and expression of specific laminin receptors, and has been found to correlate with the malignant characteristics of tumor cells (28). The adhesion of cells to laminin-1 induces collagenase IV production, which is required for tumor cells to metastasize. Previous studies, as well as the present study, have demonstrated that the adherent subclone (MH), isolated from the MKN-45 gastric cancer cell line by in vitro adhesion selection on laminin-1, is more invasive and metastatic in vitro and in vivo compared with parental (M0) and non-adherent subclone (ML) cells, indicating that the properties of these cells are closely associated with adhesion to the basement membrane and extracellular matrix (12). In addition, other studies have demonstrated that a laminin-adhesive subclone of a human colon cancer cell line revealed higher invasive and metastatic potentials in vitro and in vivo than a laminin-non-adhesive cell line. These observations indicate that the increased invasive and metastatic potentials of the laminin adhesion-selected subpopulation may be due to an alteration in the membrane distribution and/or affinities of multiple laminin receptors, including the $67 \mathrm{kDa}$ laminin receptor and $\beta 1$ integrin $(29,30)$. The cancer cell population, either as a solid tumor mass in vivo or as a continuous cell line in vitro, is an ever-changing entity due to their genetic instability and selective environmental pressure (31). Tiam-1 expression is associated with the invasive potential of gastric cancer cells. Therefore, we hypothesized that Tiam-1 expression, the rearrangement of cytoskeletal structure and the expression and/or redistribution of cell surface adhesion molecules are linked. Additionally, the associated mechanisms of cellular signal transduction remain unclear and require investigation.

Results of the present study demonstrated that Tiam-1 induces gastric cancer cell invasion and may represent a candidate target for biotherapy. However, additional studies are required to determine the molecular and biological mechanisms associated with these observations.

\section{Acknowledgements}

The authors thank Professor Zheng Jiang and his personnel at the Molecular Biological Center of Xinan Hospital (Chongqing, China) for their excellent technical assistance.

\section{References}

1. Mahar AL, McLeod RS, Kiss A, Paszat L and Coburn NG: A systematic review of the effect of institution and surgeon factors on surgical outcomes for gastric cancer. J Am Coll Surg 214: 860-868, 2012.

2. Lee JH, Kim KM, Cheong JH and Noh SH: Current management and future strategies of gastric cancer. Yonsei Med J 53: 248-257, 2012.

3. Lin LL, Huang HC and Juan HF: Discovery of biomarkers for gastric cancer: a proteomics approach. J Proteomics 75: 3081-3097, 2012. 
4. Habets GG, Scholtes EH, Zuydgeest D, van der Kammen RA, Stam JC, Berns A and Collard JG: Identification of an invasion-inducing gene, Tiam-1, that encodes a protein with homology to GDP-GTP exchangers for Rho-like proteins. Cell 77: 537-549, 1994.

5. Habets GG, van der Kammen RA, Jenkins NA, Gilbert DJ, Copeland NG, Hagemeijer A and Collard JG: The invasion-inducing TIAM1 gene maps to human chromosome band 21q22 and mouse chromosome 16. Cytogenet Cell Genet 70: 48-51, 1995.

6. Mertens AE, Roovers RC and Collard JG: Regulation of Tiam1-Rac signalling. FEBS Lett 546: 11-16, 2003.

7. Minard ME, Kim LS, Price JE and Gallick GE: The role of the guanine nucleotide exchange factor Tiam1 in cellular migration, invasion, adhesion and tumor progression. Breast Cancer Res Treat 84: 21-32, 2004

8. Chen B, Ding Y, Liu F, et al: Tiam1, overexpressed in most malignancies, is a novel tumor biomarker. Mol Med Rep 5: 48-53, 2012

9. Ding Y, Chen B, Wang S, et al: Overexpression of Tiam1 in hepatocellular carcinomas predicts poor prognosis of $\mathrm{HCC}$ patients. Int J Cancer 124: 653-658, 2009.

10. Minard ME, Ellis LM and Gallick GE: Tiam1 regulates cell adhesion, migration and apoptosis in colon tumor cells. Clin Exp Metastasis 23: 301-313, 2006.

11. Zhu JM, Yu PW and Zhao YL: Relationship between the expression of Tiam-1, Rac 1 and the pathobiological behavior of gastric cancer. Chin J Gen Surg 14: 168-172, 2005.

12. Chen XR, Ren WP, Dong JF, Xiao SD and Sloane BF: Screening of gastric cancer cell sublines by adhesion method in vitro. Chin J Gastroenterol 2: 121-124, 2001 .

13. Yokozaki H: Molecular characteristics of eight gastric cancer cell lines established in Japan. Pathol Int 50: 767-777, 2000.

14. Li ZZ, Zhang L, Mao HT, Wang Y, Li DH and Gui SL: Effect of Tiam-1 antisense oligodeoxynucleotides (ASODNS) on antimetastasis of tumor. Chin J Cancer Biother 50: 767-777, 2000.

15. Zhang XQ, Zhang R, Gu CH and Wang YM: Expression level of ICAM-1 on cultured cells measured quantitatively with cellular ELISA. Acta Acad Med Mil Tert 23: 117-118, 2001.

16. Zhang JH, Ding YQ and Yang XJ: Introduction of a reforming staining method for cytoskeletal proteins. J Diag Pathol 3: 235-236, 1996.

17. Albini A, Iwamoto Y, Kleinman HK, Martin GR, Aaronson SA, Kozlowski JM and McEwan RN: A rapid in vitro assay for quantitating the invasive potential of tumor cells. Cancer Res 47: 3239-3245, 1987.
18. Lleonart ME, Martin-Duque P, Sanchez-Prieto R, Moreno A and Ramon y Cajal S: Tumor heterogeneity: morphological, molecular and clinical implications. Histol Histopathol 15: 881-898, 2000

19. Marian AJ: Molecular genetic studies of complex phenotypes. Transl Res 159: 64-79, 2012.

20. Prasun P, Pradhan M and Agarwal S: One gene, many phenotypes. J Postgrad Med 53: 257-261, 2007

21. Etienne MS and Hall A: Rho GTPases in cell biology. Nature 420: 629-635, 2002.

22. Malliri A and Collard JG: Role of Rho-family proteins in cell adhesion and cancer. Curr Opin Cell Biol 15: 583-589, 2003.

23. Hirohashi S and Kanai Y: Cell adhesion system and human cancer morphogenesis. Cancer Sci 94: 575-581, 2003.

24. Ben-Ze'ev A: The cytoskeleton in cancer cells. Biochim Biophys Acta 780: 197-212, 1985.

25. Ehler E, van Leeuwen F, Collard JG and Salinas PC: Expression of Tiam-1 in the developing brain suggests a role for the Tiam-1-Rac signaling pathway in cell migration and neurite outgrowth. Mol Cell Neurosci 9: 1-12, 1997.

26. Okegawa T, Pong RC, Li Y and Hsieh JT: The role of cell adhesion molecule in cancer progression and its application in cancer therapy. Acta Biochim Pol 51: 445-457, 2004

27. Marhaba R and Zoller M: CD44 in cancer progression: adhesion, migration and growth regulation. J Mol Histol 35: 211-231, 2004

28. Ekblom P, Lonai P and Talts JF: Expression and biological role of laminin-1. Matrix Biol 22: 35-47, 2003.

29. Kim WH, Lee BL, Jun SH, Song SY and Kleinman HK: Expression of $32 / 67-\mathrm{kDa}$ laminin receptor in laminin adhesion-selected human colon cancer cell lines. Br J Cancer 77: 15-20, 1998.

30. Kim WH,Jun SH, Kibbey MC, Thompson EW and Kleinman HK: Expression of beta 1 integrin in laminin-adhesion-selected human colon cancer cell lines of varying tumorigenicity. Invasion Metastasis 14: 147-155, 1994-1995.

31. Brábek J, Mierke CT, Rösel D, Veselý P and Fabry B: The role of the tissue microenvironment in the regulation of cancer cell motility and invasion. Cell Commun Signal 8: 22, 2010. 